OBESITY

\title{
Neurotensin inhibition prevents weight gain
}

The development of novel anti-obesity therapies, capable of maintaining long-term weight loss without side effects, remains a global priority. Now, writing in Nature, Li, Evers and colleagues report that inhibition of the intestinal peptide neurotensin (NT) prevents the metabolically deleterious effects of a high-fat diet (HFD) in mice.

NT is a 13 -amino-acid peptide located in enteroendocrine cells of the small intestine, where it is released by fat ingestion to facilitate fatty acid absorption. High levels

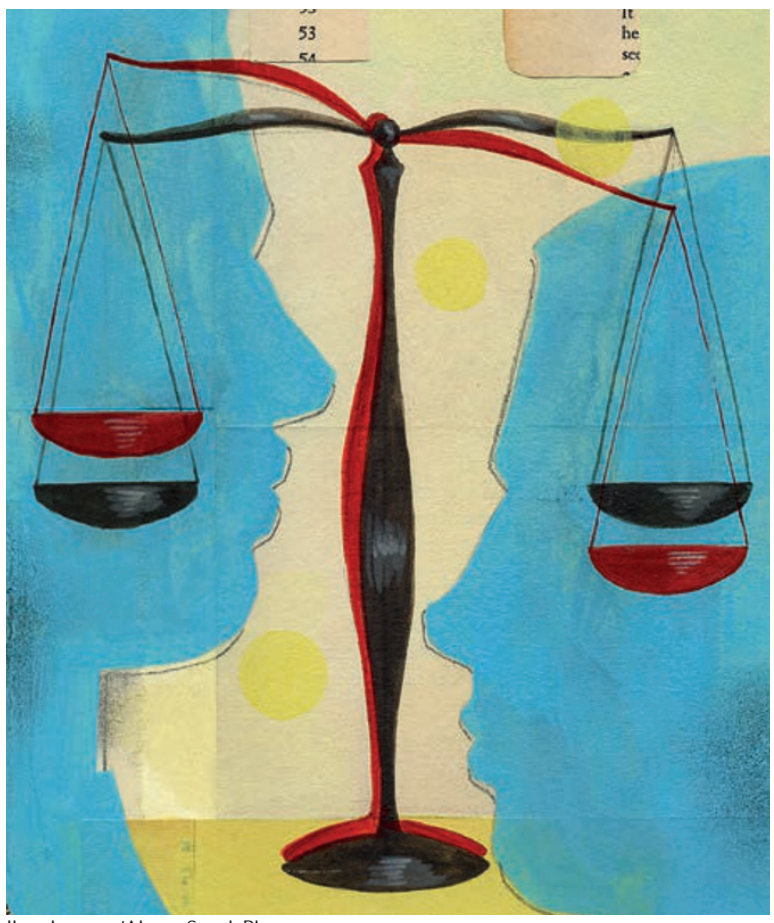

Ikon Images/Alamy Stock Photo of a stable NT precursor fragment, pro-NT, have previously been associated with increased risk of diabetes, cardiovascular disease and death, although a role for NT as a causative factor in these diseases is unknown.

To further investigate the action of NT, Evers and colleagues studied NT-deficient $\left(\mathrm{Nt}^{-1-}\right)$ mice fed a HFD for 22 weeks. They found that body weights and fat deposits of $\mathrm{Nt}^{-/-}$mice were significantly lower than those of wild-type mice. This resulted in attenuated development of obesityassociated insulin resistance, with $\mathrm{Nt}^{-/-}$mice displaying lower levels of fasting plasma glucose and insulin, greater insulin sensitivity and faster glucose clearance. Furthermore, hepatic steatosis, liver triglyceride (TG) and cholesterol accumulation were significantly decreased in $\mathrm{Nt}^{-/-}$mice, and the size and inflammatory infiltration of adipocytes in their epididymal fat pads was reduced.

The beneficial metabolic effects observed in $\mathrm{Nt}^{-1-}$ mice were not due to effects on feeding behaviour or energy expenditure but were a result of decreased intestinal lipid absorption. Importantly, there was no sign of steatorrhoea in mice deficient in NT.

Similarly, inhibition of NT activity with SR48692 - a selective antagonist of the NT receptor NTR1 - decreased intestinal fatty acid absorption in wild-type mice following olive oil gavage. In addition, treatment of wild-type mice with oral SR48692 for 13 weeks significantly attenuated body weight gain on a HFD.

Further studies in mice revealed that NT may be acting through suppression of activity of 5'-AMPactivated protein kinase (AMPK), a key fuel-sensing enzyme and critical regulator of metabolism. Indeed, levels of phosphorylated AMPK (pAMPK) were increased in the proximal intestinal mucosa of $N t^{-1-}$ mice compared to that of wildtype mice. In addition, decreased intestinal pAMPK levels observed in wild-type mice subjected to olive oil gavage were prevented by SR48692 pretreatment.

Consistent with the findings in mice, expression of human fulllength NT cDNA in Drosophila midgut enteroendocrine cells increased lipid accumulation and total body TG levels. These effects were enhanced on a HFD and were mediated by decreased gut AMPK activation.

Finally, analysis of fasting plasma concentrations of pro-NT from 4,632 middle-aged people from the population-based Malmö Diet and Cancer Study Cardiovascular Cohort revealed elevated plasma concentrations of pro-NT in obese and insulin-resistant individuals; and in non-obese individuals, high levels of pro-NT denoted a doubling of the risk of developing obesity later in life.

In summary, these findings demonstrate a key role of NT in HFD-induced obesity, highlighting NT as a prognostic marker of future obesity and a potential anti-obesity therapeutic target.

Sarah Crunkhorn

ORIGINAL ARTICLE Li, J. et al. An obligatory role for neurotensin in high-fat-diet induced obesity. Nature 533, 411-415 (2016) 